\title{
Superfluidity of a nonequilibrium Bose-Einstein condensate of polaritons
}

\author{
Michiel Wouters and Vincenzo Savona \\ Insitute of Theoretical Physics, Ecole Polytechnique Fédérale de Lausanne (EPFL), CH-1015 Lausanne, Switzerland \\ (Received 5 October 2009; revised manuscript received 17 December 2009; published 12 February 2010)
}

\begin{abstract}
We study theoretically superfluidity in a driven-dissipative Bose gas out of thermal equilibrium, and discuss the relation with conventional superfluids. We show how the superfluid behavior is characterized by a dramatic increase in the lifetime of a quantized vortex and point out the influence of the spatial geometry of the condensate. We apply our study to a condensate of polaritons in a semiconductor microcavity, whose properties can be directly inferred from optical spectroscopy. We propose three different experimental schemes to measure the vorticity of the polariton condensate.
\end{abstract}

DOI: 10.1103/PhysRevB.81.054508

PACS number(s): 67.10.-j, 03.75.Kk, 05.70.Ln, 71.36.+c

\section{INTRODUCTION}

The connection between the two intriguing phenomena of superfluidity and long-range spatial coherence was conjectured soon after the discovery of superfluidity. It has proven to be subtle, but is now well established for Bose-Einstein condensates at thermal equilibrium. ${ }^{1}$ Not all degenerate Bose gases of interest are, however, at thermodynamical equilibrium. For example, photon lasers, atom lasers, ${ }^{2}$ and BoseEinstein condensates of microcavity polaritons ${ }^{3-5}$ are drivendissipative systems that show deviations from thermal equilibrium, yet they undergo a transition to a coherent state above a critical density. The question then naturally arises whether these nonequilibrium systems display superfluidity in some sense. Phenomenologically, superfluidity can be loosely described as "flow without friction," referring to the remarkable property that the superfluid does not necessarily move along with the container that holds it. This idea of flow without friction does not straightforwardly apply to drivendissipative systems: when energy is fed into the system, it does not come as a surprise that the fluid is in motion with respect to its container. A slightly more general view on superfluidity is that it allows for many metastable flow patterns under the same boundary conditions. Such behavior is not displayed by classical systems: the velocity of water in a pipe with a given pressure difference between both ends converges quickly to its unique steady state. The definition of superfluidity as multiple metastable flow patterns is therefore equally meaningful in nonequilibrium systems.

A prototypical system where these issues can be investigated is a polariton condensate. Recent experiments have evidenced the quantum collective behavior of exciton polaritons in semiconductor microcavities, through the observation of the spontaneous buildup of long-range spatial coherence ${ }^{3}$ as well as a bimodal momentum distribution ${ }^{3-5}$ and long temporal coherence. ${ }^{6}$ Due to the short polariton lifetime, the polariton gas has to be continuously replenished by means of relaxation from an excitonic reservoir that is excited by a laser. An insightful approach toward superfluidity in nonequilibrium polariton condensates is the study of vorticity. In Ref. 7, where quantized vortices were observed, it was, however, pointed out that the quantized vorticity cannot be directly seen as a proof of superfluidity, but rather as a flow that results from the interplay between the exciton relaxation and the shape of the potential landscape acting on the polaritons. The same holds for the spontaneously formed vortex lattice that was predicted in the theoretical work of Ref. 8.

The first experimental sign of superfluidity in semiconductor microcavities was the ballistic propagation of polariton bullets that was observed by Amo et al. ${ }^{9}$ These bullets are introduced by a probe pulse and propagate over large distances without showing dissipation. Additionally, in a resonantly driven polariton gas where the coherence is imprinted by the excitation laser, superfluidity manifests itself as a suppression of the Rayleigh scattering below the critical velocity. ${ }^{10,11}$

Experiments that investigate metastable flow patterns have recently been carried out on a triggered optical parametric oscillator, where Sanvitto et al. ${ }^{12}$ used a probe that carries angular momentum to create a quantum degenerate polariton fluid on top of the spontaneous optical parametric oscillator signal. In this setting, it was observed that the vortex lifetime is as long as the lifetime of the quantum fluid that is formed by the additional signal density induced by the probe.

In this paper, we use a stochastic classical field mode ${ }^{13}$ to study the superfluid nature of a spontaneously formed polariton condensate through the analysis of the vorticity induced by a probe with finite angular momentum. In contrast to the recent experiments, ${ }^{12}$ we find that the vorticity can be fully sustained by the spontaneous polariton condensate and its lifetime is expected to be much longer than the perturbation in the density induced by the probe. In this respect, the presence of a vortex represents another metastable solution other than the zero angular-momentum condensate.

In this scenario, fluctuations play an important role. They determine the presence or absence of spatial long-range order characterized by the spatial coherence function $g^{(1)}\left(\mathbf{x}, \mathbf{x}^{\prime}\right)$. At equilibrium, the presence of long-range spatial coherence is a necessary condition for the appearance of superfluidity. ${ }^{1}$ In the superfluid state, fluctuations still induce a random motion of the vortex core. Because it is at present technologically impossible to perform single shot measurements, direct visualization of a moving vortex in the density or phase profile of the condensate can therefore not be achieved. We will propose instead three different operational methods to detect the condensate vorticity in multiple shot measurements.

We describe our model for polariton condensation in Sec. II. The angular momentum and vortex motion are analyzed 
in Sec. III. The problem of detecting angular momentum is addressed in Sec. IV. The conclusions are drawn in Sec. V.

\section{MODEL}

Our classical field model describes the dynamics of a classical incoherent exciton reservoir coupled to the quantum dynamics in the lower polariton branch. When accounting for the polarization degree of freedom, vorticity becomes a very rich subject. ${ }^{14}$ In order to restrict to a single polarization state, the degeneracy between TE/TM modes can be split by the anisotropy of the microcavity. ${ }^{15}$ Alternatively, a magnetic field could be applied to favor relaxation toward a welldefined, circularly polarized eigenstate. Therefore, we will address the simpler case of a scalar field. The equation of motion of the reservoir describes the nonresonant excitation with intensity $P$, and the scattering into the lower polariton region

$$
\begin{aligned}
\frac{d}{d t} \tilde{n}_{R}= & P-\gamma_{R} \tilde{n}_{R}-\left.\alpha \frac{d}{d t}\left\langle\psi^{\dagger} \psi\right\rangle\right|_{\text {res }}-\frac{\alpha}{2 \Delta V} \sum_{\mathbf{k}}\left\{\widetilde{n}_{R}^{2} R_{\text {in }}\left[\epsilon_{L P}(\mathbf{k})\right]\right. \\
& \left.-\tilde{n}_{R} R_{\text {out }}\left[\epsilon_{L P}(\mathbf{k})\right]\right\},
\end{aligned}
$$

where $R_{\text {in(out) }}$ quantifies the scattering rate from the reservoir (lower polariton branch) into the lower polariton branch (reservoir). The energy dependence of the rates is evaluated on the bare dispersion of the lower polariton branch $\epsilon_{L P}(\mathbf{k})$ $=k^{2} / 2 m_{L P}$, with $m_{L P}$ the effective lower polariton mass. The reservoir density $\widetilde{n}_{R}$ is normalized so that threshold is reached when it is unity. The lower polariton dynamics is described by the stochastic classical field equation

$$
\begin{aligned}
i d \psi= & d t\left[\frac{-\hbar^{2} \nabla^{2}}{2 m_{L P}}+\frac{i\left(\mathcal{R}_{\text {in }}-\mathcal{R}_{\text {out }}-\gamma\right)}{2}+\frac{g}{\Delta V}|\psi|^{2}\right] \psi \\
& +F_{p} e^{-i \omega_{p} t} d t+d W .
\end{aligned}
$$

Here $\Delta V$ is the volume of the elementary cell on which the lower polariton field is discretized, $F_{p}$ and $\omega_{p}$ are the amplitude and frequency of an additional resonant laser that excites the microcavity. The noise term $d W$ is a complex Gaussian stochastic variable with the correlation functions

$$
\begin{gathered}
\left\langle d W(\mathbf{x}) d W\left(\mathbf{x}^{\prime}\right)\right\rangle=0, \\
\left\langle d W(\mathbf{x}) d W^{*}\left(\mathbf{x}^{\prime}\right)\right\rangle=\frac{d t}{4 \Delta V}\left(\left\langle\mathbf{x}\left|\mathcal{R}_{\text {in }}^{S}+\mathcal{R}_{\text {out }}^{S}\right| \mathbf{x}^{\prime}\right\rangle+2 \gamma \delta_{\mathbf{x}, \mathbf{x}^{\prime}}\right),
\end{gathered}
$$

where $\mathcal{R}_{\text {in,out }}^{S}=\left[\mathcal{R}_{\text {in,out }}+\left(\mathcal{R}_{\text {in,out }}\right)^{\mathrm{T}}\right] / 2$ are the symmetrized inand out-scattering kernels, defined as ${ }^{16}$

$$
\mathcal{R}_{\text {in,out }} \psi(\mathbf{x})=\sum_{\mathbf{q}, \mathbf{x}^{\prime}} e^{i \mathbf{q}\left(\mathbf{x}-\mathbf{x}^{\prime}\right)} \sqrt{n_{R}(\mathbf{x}) n_{R}\left(\mathbf{x}^{\prime}\right)} R_{\text {in,out }}[\boldsymbol{\epsilon}(\mathbf{q})] \psi\left(\mathbf{x}^{\prime}\right) .
$$

According to Eq. (2), the number of excitons that scatter from the reservoir into the lower polariton classical field equals $\left.\frac{d}{d t}\left\langle\psi^{\dagger} \psi\right\rangle\right|_{\text {res }}=2 \operatorname{Re}\left[\psi^{*}\left(\mathcal{R}_{\text {in }}-\mathcal{R}_{\text {out }}\right) \psi\right]$. The parameter $\alpha$ in Eq. (1) quantifies the strength of the coupling of the reservoir density to the lower polariton field $\psi$; the last term in Eq. (1) comes from the fact that the classical field in Eq. (2) contains 1/2 zero-point fluctuations per mode, because it samples the Wigner quasiprobability distribution $P_{W}$, that is related to the observable density as $\int P_{W}(\psi) \psi^{*} \psi$ $=\left\langle\hat{\psi} \hat{\psi}^{\dagger}+\hat{\psi} \hat{\psi}^{\dagger}\right\rangle / 2$. In the simulations, we have used the following parameters: $\quad \gamma=0.5 \mathrm{meV}, \quad m_{L P} / \hbar$ $=1 \mu \mathrm{m}^{-2} \mathrm{meV}^{-1}, g / \hbar=0.005 \mathrm{meV} \mu \mathrm{m}^{2}, R_{\text {out }}(E)=3 \mathrm{meV}$, $R_{\text {in }}(E)=\exp \left(-E / k_{B} T_{R}\right)[\gamma+3 \mathrm{meV}]$ with $k_{B} T_{R}=2 \mathrm{meV} . \mathrm{Nu}-$ merical results are obtained by averaging over 100 realizations of the noise.

\section{METASTABLE VORTICITY}

Vortices are typically more stable in a toroidal geometry as compared to a simply connected one. So to create a ringshaped condensate, one can use the fact that the condensate shape follows the shape of the excitation laser. We therefore start the analysis of the vortex lifetime for a polariton gas that is excited by a ring-shaped pump laser. The right-hand panels in Fig. 1 show the simulated evolution of the angular momentum and excess density when a Gauss-Laguerre beam with one unit of angular momentum is applied to the polariton gas for three different condensate densities (increasing from top to bottom). The corresponding spatial coherence before the arrival of the probe pulse is shown in the left-hand panels. The excess density (thin dashed line) evolves almost identically in the three cases, ${ }^{17}$ but the time dependence of the angular momentum is dramatically different. At low densities, the angular momentum quickly decays together with the excess density introduced by the probe pulse, whereas at the highest density, the angular momentum lasts up to the end of our simulation time window $(120 \mathrm{ps})$. The insets in the left column show the spatial coherence at the final time of our simulation window. In the upper and central panels, where the vortex disappears at the final time of the simulations, an important decrease in the absolute value of the coherence is observed.

The simultaneous increase in the coherence and the vortex lifetime is analogous to the case of conventional superfluids. The argument for the difference in lifetime of the angular momentum is that when the phase of the condensate is coherent over large distances, a local distortion of the phase is not possible and vorticity can only disappear if the vortex core moves out of the polariton gas. The time it takes for a vortex to move out depends on the geometry of the condensate. In the well-condensed regime, it is meaningful to study the statistics of the vortices in the individual Monte Carlo simulations of the polariton field (in the incoherent regime, there are many spontaneous vortex-antivortex pairs that make this analysis meaningless). In the lower right panel of Fig. 1, we show the probability to have a vortex within the condensate area together with the angular-momentum evolution. Initially, this probability is one, because the vortex is imprinted by the strong probe pulse. Up to about 200 ps, this probability remains close to unity. After 200 ps, the vortex probability also starts to decrease, because of the finite escape rate of vortices out of the center of the ring. Note that 

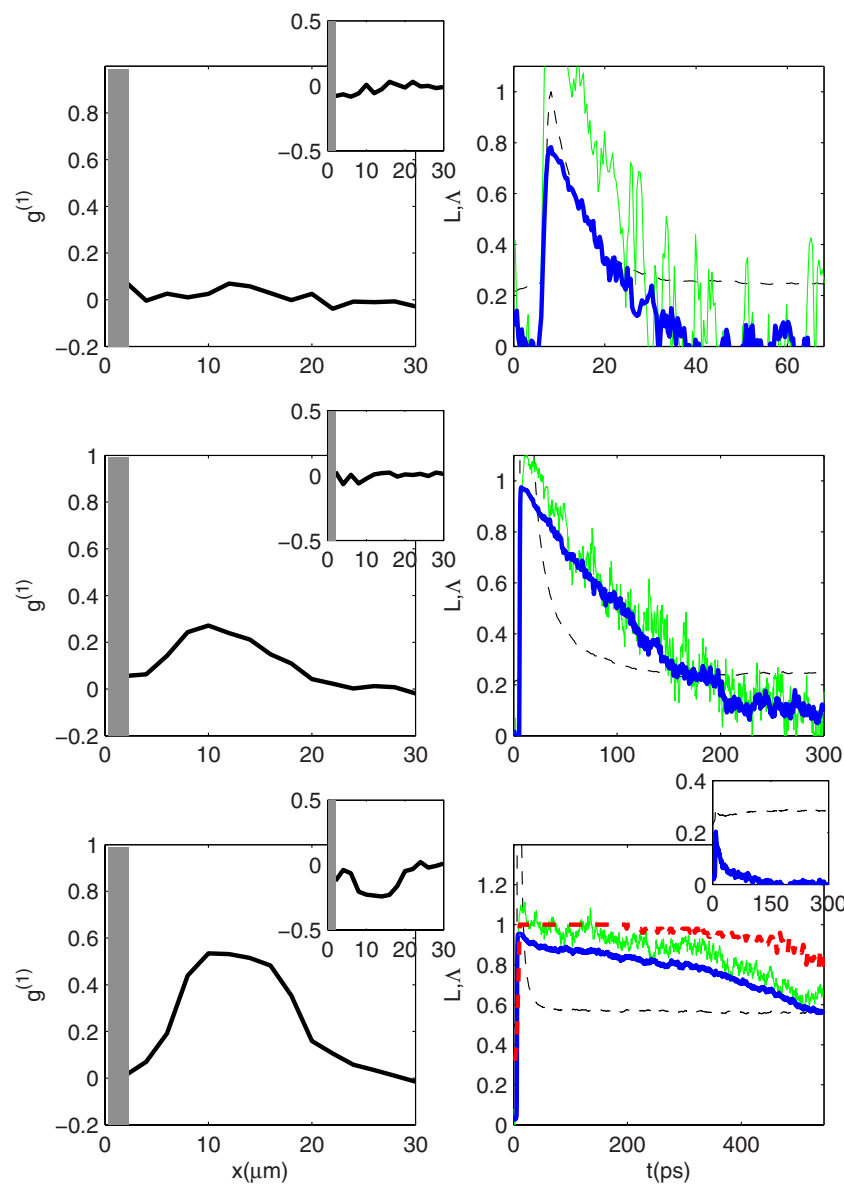

FIG. 1. (Color online) The left column show the first-order spatial coherence between inversion symmetric points $g^{(1)}(x)$ $=\left\langle\psi^{\dagger}(-\mathbf{x}) \psi(\mathbf{x})\right\rangle / \sqrt{n(\mathbf{x}) n(-\mathbf{x})}$ for values of the density increasing from top to bottom ( $n=1.3,5.7$, and $32 \mu \mathrm{m}^{-2}$, respectively), before the arrival of the Gauss-Laguerre pulse; the insets show the values at the latest simulated times. The right column shows the temporal evolution of the total polariton density (thin dashed line) and angular momentum (thick full line) after a 1 ps Gauss-Laguerre probe pulse with a waist of $20 \mu \mathrm{m}$ has perturbed the polariton condensates. The additional density introduced by the resonant probe pulse is, from top to bottom, four, forty, and twenty times the condensate density. The thin line shows the observable $\Lambda$ that we propose to use for a time-resolved measurement of the vorticity. The thick dashed line shows the probability that the vortex core is still inside the condensate region. The inset in the lower right figure shows the response of the condensate to a probe pulse that is too weak to create a vortex. The polariton condensate is created with a ringshaped excitation laser (inner radius $7 \mu \mathrm{m}$ and outer radius $20 \mu \mathrm{m})$.

the onset of vortex escape is reflected in a fastening of the angular-momentum decay.

In polariton condensates, an important phenomenon that determines the lifetime of the superflow are the currents that arise due to the interplay between the nonresonant excitation and the external potential. ${ }^{18}$ Vortices are dragged by these currents and in order to achieve a long lifetime of the vortex it is beneficial if these currents are pointed toward the center of the vortex. Under excitation with a ring-shaped excitation laser of Fig. 1, the blueshift causes the polaritons to flow
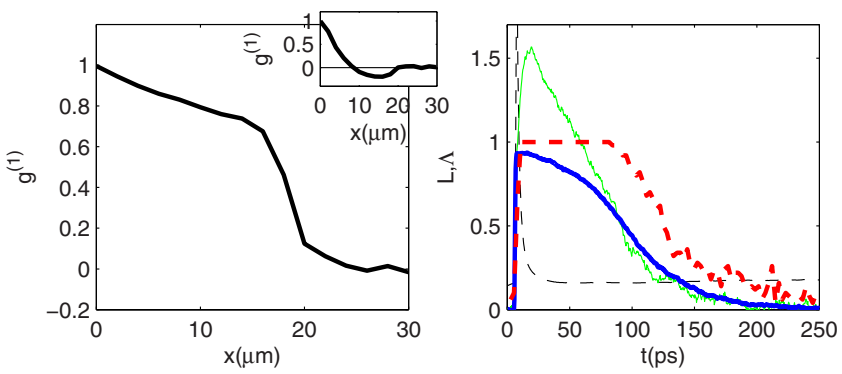

FIG. 2. (Color online) The same as Fig. 1, but for a condensate excited with a top-hat excitation laser with a radius of $20 \mu \mathrm{m}$. The density in the center is $n=48 \mu \mathrm{m}^{-2}$. The inset in the left-hand panel shows the spatial coherence at $t=100 \mathrm{ps}$. The pump intensity is the same as in the lower panels of Fig. 1.

toward the center, which hinders the vortex to leave the system, because it has to move upstream. Figure 2 shows the same simulations with a regular top hat excitation laser. The polariton-polariton interactions now cause an outward instead of inward flow and the angular momentum in the phase coherent regime decays much quicker.

Some representative trajectories of the vortex motion are plotted in Fig. 3. At short times, the vortex undergoes a random motion close to the center of the condensate. When its distance from the center increases, we observe that the path has a higher probability of going out of the condensate. This typically occurs in a slightly spiraling motion, familiar from atomic BECs. ${ }^{19}$ The corresponding angular momentum is shown in the right-hand panels. When the vortex leaves the condensate (vertical line), the angular momentum becomes very small. The most important loss mechanism of angular momentum is therefore the escape of the vortex out of the condensate. During the time the vortex spends within the polariton gas, the angular momentum in the reference frame of the vortex core remains unity as well. The random fluctuations in the position of the vortex core can, however, not be followed in experiments. Therefore, we plot the angular momentum with respect to the center of the polariton cloud. This angular momentum decreases when the vortex moves out of the center. It is important to note that this decrease in angular momentum is not related to a loss of superfluidity, but is merely due to the finite size of the polariton cloud.

Different vortex trajectories are found under excitation with a ring-shaped laser. The upper panel of Fig. 4 shows a case where the vortex motion is confined by the high-density region in the pumped region and the value of angular momentum stays close to one. Most of the trajectories in the high-density regime are of this kind. The lower panel shows the more rare case where the vortex leaves the inner part of the ring, with a decay of the angular momentum as a result.

The phase rigidity of the polariton condensate becomes also clear in the response of the angular momentum for varying intensity of the Gauss-Laguerre pulse. At low intensities, the pulse is not able to create a vortex in the condensate so that the transfer of angular momentum is poor and decays quickly. Only when a certain threshold intensity is passed (corresponding in our simulations to an excess density of about half the condensate density) a vortex is formed and the angular momentum persists for long times. This behavior is 

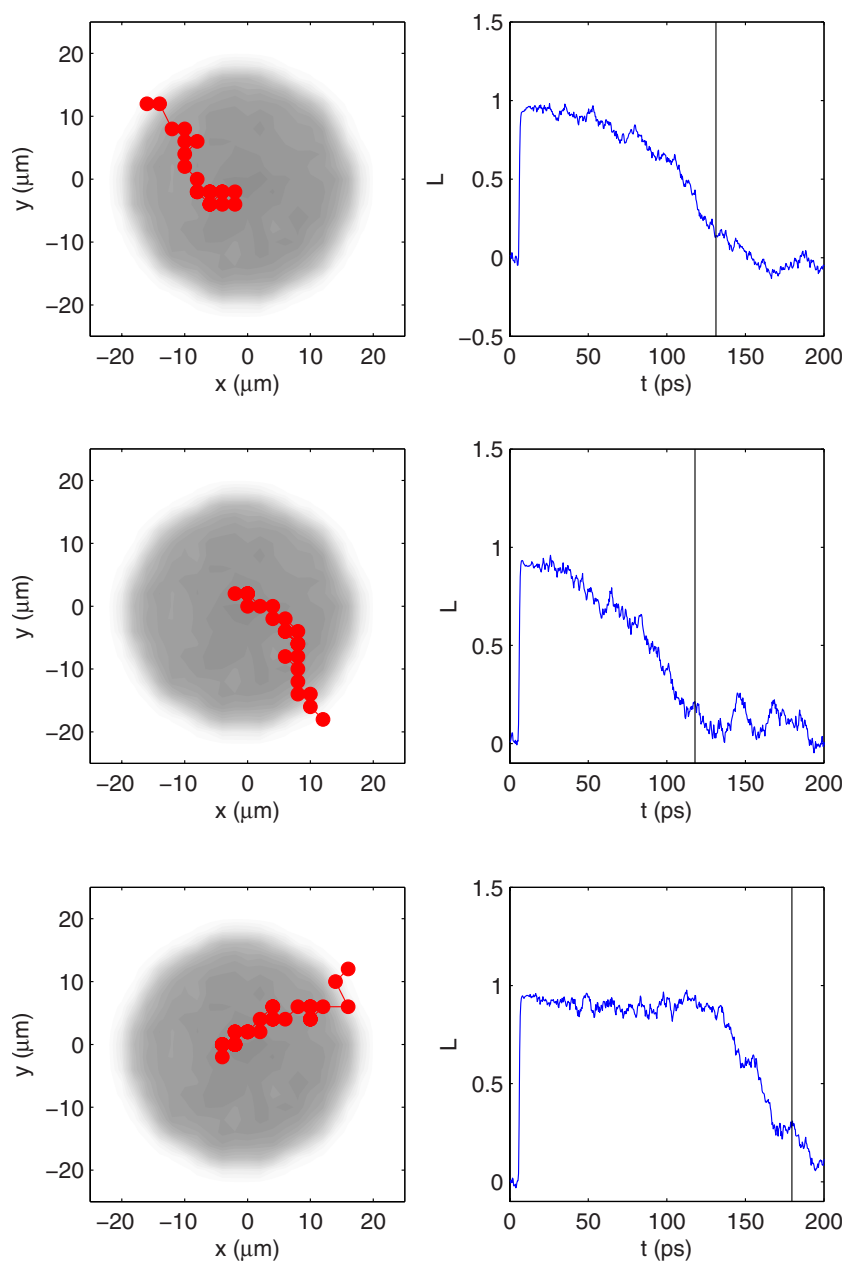

FIG. 3. (Color online) Representative vortex trajectories (lefthand panels) for the top hat condensate from Fig. 2 on top of the polariton density (in gray scale). The right-hand panels show the angular momentum of the corresponding Monte Carlo simulation. The vertical line shows the time when the vortex leaves the condensate. Typically, the angular momentum is very close to zero when the vortex leaves.

illustrated in the inset in the lower right panel of Fig. 1, where the response to a low-intensity pulse is shown. In the normal, incoherent phase, we have observed no qualitative change in the transfer of angular momentum: it always decays on the same time scale as the excess density introduced by the pulse.

\section{VORTEX DETECTION SCHEMES}

We now turn to the question how the metastable superflow can be detected experimentally. Ideally, an experimental measurement should be time resolved, so as to observe the predicted increase in lifetime of the metastable superflow when the condensate density is increased. Because experiments cannot resolve single shot condensate dynamics on the picosecond time scale, the random position of the vortex core makes the vortex detection nontrivial. Two of the three proposals below look at vortex signatures within a finite-time window at points in space that have not been reached by the
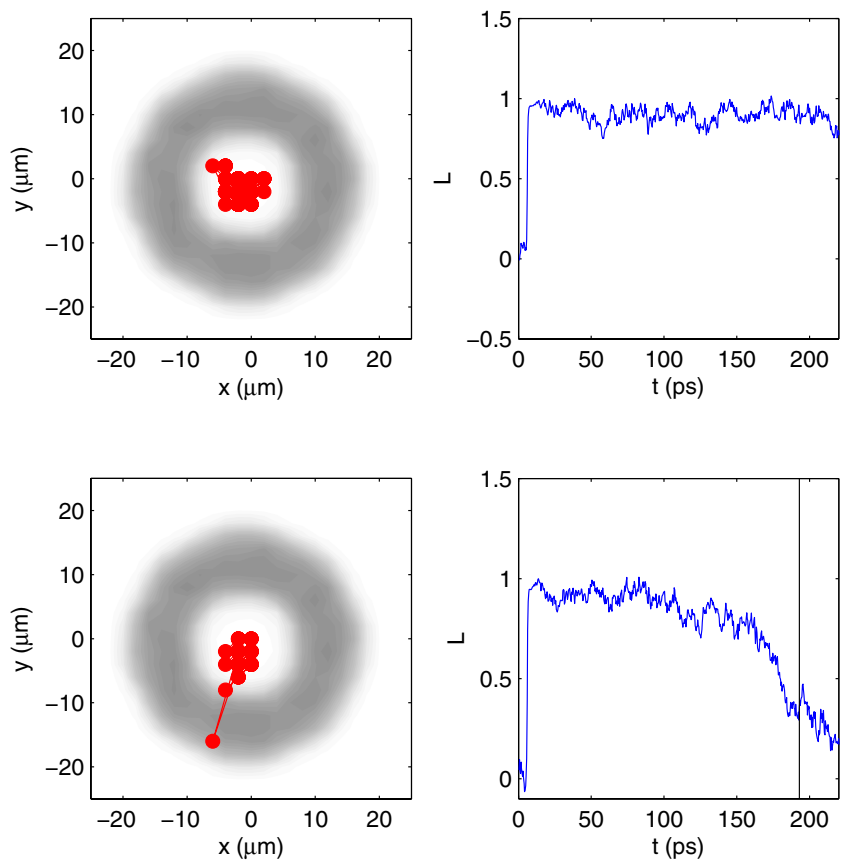

FIG. 4. (Color online) The same as in Fig. 3, but under excitation with a ring-shaped laser spot.

vortex core during this time. The third interferometric proposal on the other hand relies on a spatially extensive feature.

The first technique that we suggest is the one that was used previously to detect vortices induced by the disordered potential acting on the polaritons. A vortex leads to a fork dislocation in the interference pattern of the condensate with its inverted copy. ${ }^{7}$ In order to observe the increased lifetime of the superflow when the condensate density is increased, this interferogram should be measured with time resolution. This is possible with the use of a streak camera. Still, an average over many runs of the experiment should be recorded in order to have a good signal to noise ratio. This poses some difficulties when the vortex undergoes a random motion that is different from shot to shot. In that case, no dislocation will be visible in an interferogram that is averaged over many realizations.

A first possibility to do a time-resolved measurement is based on the change in sign of $g^{(1)}(\mathbf{x},-\mathbf{x})$ (compare the lefthand plots in Fig. 1 with their insets). In a time-resolved interferogram, such as the one recorded in Ref. 20, this would be visible as an interchange between light and dark fringes as a function of time. The farther apart the points between which the spatial coherence is measured, the longer is the time the phase change remains visible. This feature is clearly visible in the inset of Fig. 2, where the phase shift only persists for points that are more than $10 \mu \mathrm{m}$ away from the center. The reason is that there is a large phase difference only in points $\mathbf{x}$ and $\mathbf{x}^{\prime}$ that are farther away from the center than the average distance the vortex has traveled. Note that the shape of $g^{(1)}(\mathbf{x},-\mathbf{x})$ close to $\mathbf{x}=0$ implies that no fork dislocation is present in the interference pattern.

A second possibility is a direct measurement of the time dependence of the angular momentum. We discuss here how 

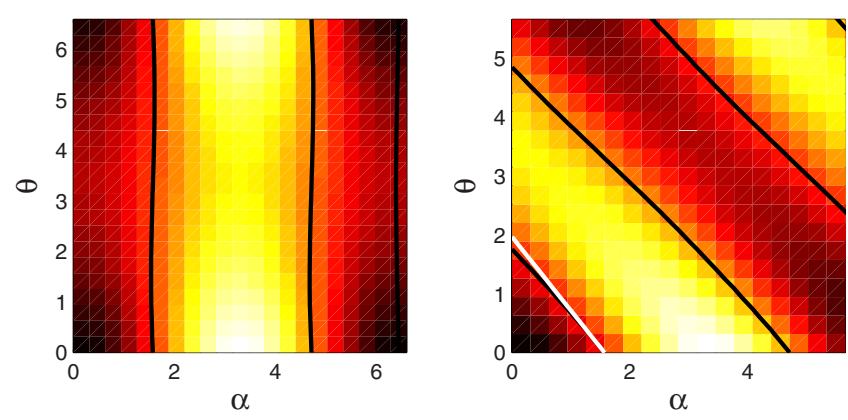

FIG. 5. (Color online) Interferogram as a function of the phase difference $\alpha$ and rotation angle $\theta$ between the copies of the condensate that are interfered, before ( $t=0 \mathrm{ps}$, left-hand panel) and after $(t=250 \mathrm{ps}$, right-hand panel) the arrival of the Gauss-Laguerre pulse. The black lines show the contour lines $I(\alpha, \theta)=1$ and the white line shows $\alpha=\pi / 2+\left\langle\hat{L}_{z}\right\rangle \theta$, where $\left\langle\hat{L}_{z}\right\rangle$ is taken from Fig. 1 . The presence of angular momentum is signaled by the tilting of the interference fringes in the $(\alpha, \theta)$ plane.

this can be achieved with interferometry. The operator that rotates a state over an angle $\theta$ can be written as $\hat{R}_{\theta}$ $=\exp \left(i \theta \hat{L}_{z}\right)$. Letting the polariton condensate interfere with a copy that is rotated by an angle $\theta$ and phase shifted by $\alpha$ leads to a integrated intensity, normalized to the total number of particles $N$

$$
\begin{aligned}
I(\alpha, \theta) & =\frac{1}{2 N} \int d \mathbf{x}\left\langle\left|\hat{\psi}(\mathbf{x})+e^{i \alpha} \hat{\psi}\left(R_{-\theta} \mathbf{x}\right)\right|^{2}\right\rangle, \\
& =1-\frac{i \hbar}{N} \int d \mathbf{x}\left\langle\hat{\psi}^{\dagger}(\mathbf{x}) \cos \left[\theta(\mathbf{x} \times \nabla)_{z}+\alpha\right] \hat{\psi}(\mathbf{x})\right\rangle .
\end{aligned}
$$

For small rotation angles and $\alpha \approx \pi / 2$, the argument of the cosine can be expanded. It is then easy to see that the contour line of the interferogram $I(\alpha, \theta)=1$ goes to $\left\langle\hat{L}_{z}\right\rangle \theta=\pi / 2-\alpha$. If the fluctuations of the angular momentum are small, the contour lines of the interferogram are straight lines and the angular momentum can be determined from a large region in the $(\alpha, \theta)$ plane. Figure 5 shows simulated interferograms. Left and right panels are before and after the Gauss-Laguerre pulse, respectively. In the right-hand panel, the vorticity of the condensate results in a tilting of the interferogram in the $(\alpha, \theta)$ plane. Note the decrease in the contrast of the interferogram at finite rotation angles $\theta$. This is a consequence of the imperfect spatial coherence.

An alternative scheme that could be used to detect the presence of a vortex is based on the fact that a vortex induces

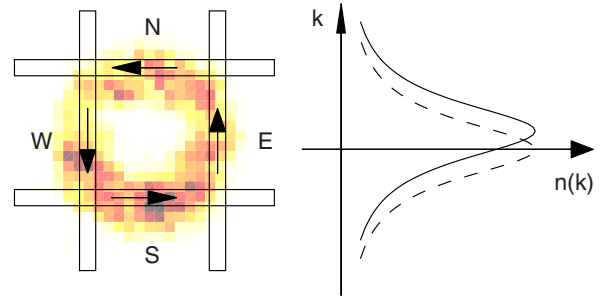

FIG. 6. (Color online) Sketch of the scheme to detect the extra velocity in the polariton condensate due to the presence of a vortex. The momentum distribution from a slice selected in real space is shifted in the presence of a vortex (full line) with respect to the momentum distribution in its absence (dashed line).

a flow of polaritons $v \propto 1_{\theta} / r$. Therefore, measuring the velocity (momentum) of polaritons on a path around the vortex can reveal its presence. The proposal to measure this velocity is sketched in Fig. 6. It consists of measuring the momentum distribution of a slice of polaritons selected in real space. The presence of vortex will be signaled by a shift in the momentum distribution along the long directions of the slits. The observable that corresponds best to the angular momentum is $\Lambda=(\pi R / 4)\left\langle k_{E}-k_{N}-k_{W}+k_{S}\right\rangle$, where $R$ is the distance from the center of the condensate to the slits. $\Lambda$ is plotted in Figs. 1 and 2 together with the angular momentum $L$, showing a good correspondence between $\Lambda$ and $L$.

\section{CONCLUSIONS}

In conclusion, we have theoretically addressed the superfluid properties of a nonequilibrium Bose-Einstein condensate of polaritons by investigating the lifetime of a quantized vortex that is introduced by a resonant laser. Only when the condensate exhibits a large degree of long-range order, the lifetime of the angular momentum substantially exceeds the lifetime of the excess density introduced by the resonant laser beam. We have pointed out the importance of the geometry of the condensate and proposed and analyzed several schemes to experimentally detect the presence of a quantized vortex.

\section{ACKNOWLEDGMENTS}

It is a pleasure to thank I. Carusotto, D. Sarchi, and K. Lagoudakis for stimulating discussions. We acknowledge the support of NCCR Quantum Photonics (NCCR QP), research instrument of the Swiss National Science Foundation (SNSF).

\footnotetext{
${ }^{1}$ A. J. Leggett, Quantum Liquids: Bose Condensation and Cooper Pairing in Condensed-Matter Systems (Oxford University Press, New York, 2006).

${ }^{2}$ A. P. Chikkatur, Y. Shin, A. E. Leanhardt, D. Kielpinski, E. Tsikata, T. L. Gustavson, D. E. Pritchard, and W. Ketterle, Science 296, 2193 (2002).
}

${ }^{3}$ J. Kasprzak, M. Richard, S. Kundermann, A. Baas, P. Jeambrun, J. M. J. Keeling, F. M. Marchetti, M. H. Szymańska, R. André, J. L. Staehli, V. Savona, P. B. Littlewood, B. Deveaud, and Le Si Dang, Nature (London) 443, 409 (2006).

${ }^{4}$ H. Deng, D. Press, S. Gotzinger, G. S. Solomon, R. Hey, K. H. Ploog, and Y. Yamamoto, Phys. Rev. Lett. 97, 146402 (2006). 
${ }^{5}$ R. Balili, V. Hartwell, D. Snoke, L. Pfeiffer, and K. West, Science 316, 1007 (2007)

${ }^{6}$ A. P. D. Love, D. N. Krizhanovskii, D. M. Whittaker, R. Bouchekioua, D. Sanvitto, S. Al Rizeiqi, R. Bradley, M. S. Skolnick, P. R. Eastham, R. André, and L. S. Dang, Phys. Rev. Lett. 101, 067404 (2008).

${ }^{7}$ K. G. Lagoudakis, M. Wouters, M. Richard, A. Baas, I. Carusotto, R. André, Le Si Dang, and B. Deveaud-Plédran, Nat. Phys. 4, 706 (2008).

${ }^{8}$ J. Keeling and N. G. Berloff, Phys. Rev. Lett. 100, 250401 (2008).

${ }^{9}$ A. Amo, D. Sanvitto, F. P. Laussy, D. Ballarini, E. del Valle, M. D. Martin, A. Lemaître, J. Bloch, D. N. Krizhanovskii, M. S. Skolnick, C. Tejedor, and L. Viña, Nature (London) 457, 291 (2009).

${ }^{10}$ I. Carusotto and C. Ciuti, Phys. Rev. Lett. 93, 166401 (2004).

${ }^{11}$ A. Amo, J. Lefrere, S. Pigeon, C. Adrados, C. Ciuti, I. Carusotto, R. Houdre, E. Giacobino, and A. Bramati, Nat. Phys. 5, 805 (2009).

${ }^{12}$ D. Sanvitto, F. Marchetti, M. Szymanska, G. Tosi, M. Baudisch, F. Laussy, D. Krizhanovskii, M. Skolnick, L. Marrucci, A. Lemaitre, J. Bloch, C. Tejedor, and L. Vina, arXiv:0907.2371 (unpublished).
${ }^{13}$ M. Wouters and V. Savona, Phys. Rev. B 79, 165302 (2009).

${ }^{14}$ Y. G. Rubo, Phys. Rev. Lett. 99, 106401 (2007).

${ }^{15}$ Ł. Kłopotowski, M. D. Martín, A. Amo, L. Viña, I. A. Shelykh, M. M. Glazov, G. Malpuech, A. V. Kavokin, and R. André, Solid State Commun. 139, 511 (2006).

${ }^{16}$ The geometric mean of $n_{R}$ at positions $\mathbf{x}$ and $\mathbf{x}^{\prime}$ is a slight modification with respect to the one that is derived from a microscopic Hamiltonian, that simplifies the generation of the noise.

${ }^{17}$ Note, however, the slower decay of the excess density when closer to threshold, related to the critical slowing down, see Ref. 21 for an experimental and theoretical discussion in the parametric oscillation regime.

${ }^{18}$ M. Wouters, I. Carusotto, and C. Ciuti, Phys. Rev. B 77, 115340 (2008).

${ }^{19}$ P. O. Fedichev and G. V. Shlyapnikov, Phys. Rev. A 60, R1779 (1999).

${ }^{20}$ G. Nardin, K. G. Lagoudakis, M. Wouters, M. Richard, A. Baas, R. André, Le Si Dang, B. Pietka, and B. Deveaud-Plédran, Phys. Rev. Lett. 103, 256402 (2009).

${ }^{21}$ D. Ballarini, D. Sanvitto, A. Amo, L. Viña, M. Wouters, I. Carusotto, A. Lemaitre, and J. Bloch, Phys. Rev. Lett. 102, 056402 (2009). 